\title{
Social and Personal Context Modeling for Contact List Recommendation on Mobile Device
}

\author{
Jun-Ki Min, Hee-Taek Kim, and Sung-Bae Cho \\ Department of Computer Science, Yonsei University, \\ 262 Seongsanno, Seodaemun-gu, Seoul 120-749, Korea \\ \{loomlike,elsein\}@sclab.yonsei.ac.kr,sbcho@cs.yonsei.ac.kr
}

\begin{abstract}
This paper presents a social and personal context modeling method on mobile device. It infers user's contexts, such as amity with others and emotional state, from uncertain logs stored in mobile devices using Bayesian networks. Proper services are then provided to the user based on the semantic compatibility between current and past contexts. Here, the contexts are hierarchically matched after each context is expanded to the context-tree using domain ontology. We have implemented a contact list recommendation application on mobile device that recommends phone numbers in a phonebook according to the user's situation. Experimental results on real-user data show that the method provides an efficient and accurate means for mobile based social networking applications.
\end{abstract}

\section{Introduction}

A social network is a set of individuals or groups connected by common features like values, visions, ideas, and friendship. Social networking has provided powerful new ways to find people, groups, communicate and share information [1]. A social network service has focused on building web based online communities because the internet provides a variety of ways for users to interact, such as e-mail and instant messaging services [2].

Recently, interest in social network and social context modeling on a mobile environment has increased rapidly since the users can share their information through person-to-person interaction with mobile devices. Lee et al. [3] developed an emoticon transformation service on mobile devices which infers the user's emotion and transfers the corresponding emoticon to other users in the network. Sadeh et al. [4] introduced an agent-based environment, called
MyCampus, where the agents access to intranet or internet services as they assist their users in carrying out different tasks such as planning an evening out or organizing a study group.

The applications presented so far, however, only consider low-level contexts which are extracted based on the rules. In this paper, we propose a social and personal context modeling method to support social networking applications of mobile devices. The proposed method uses a number of Bayesian networks (BNs) to infer the user's high-level contexts. It can efficiently handle numerous uncertainties existed in the mobile environment like inaccuracy of sensors and unknown causal factors. The proposed method then selects proper services by matching current contexts to the user's service usage history in a hierarchical manner which is able to treat the fuzziness of context information. Based on the proposed method, we introduce a smart phonebook that recommends contact list according to the user's current situation.

\section{Background}

A mobile device like PDA or smart phone can easily collect user's logs from personal information management system (PIMS), global positioning system (GPS), calling history, and short messages services (SMS). It also can be customized to fit user's preferences and can provide context-aware applications effectively because it is used in a much wider range of situations and is carried by the user at all times [5]. Context awareness, however, is not easy to achieve because of the environmental uncertainties like user's behavioral irregularity, sensors' inaccuracy, and causal uncertainty. Since BN is one of efficient tools to handle the uncertain and partial information, it has been widely used to context reasoning and modeling problems. Park et al. trained BNs using 
context information with user request gathered from the mobile device, and applied it to a map-based recommendation system [6]. Cho et al. presented the 'AniDiary' which extracts landmarks from user's daily life logs using BNs and summarizes the logs with a cartoon-style diary [7].

\section{Contact list recommendation}

In our approach the user contexts are derived from user's mobile logs using BNs, and are then matched to services in a hierarchical manner in order to treat the fuzzy characteristics of contextual information more effectively (Figure 1).

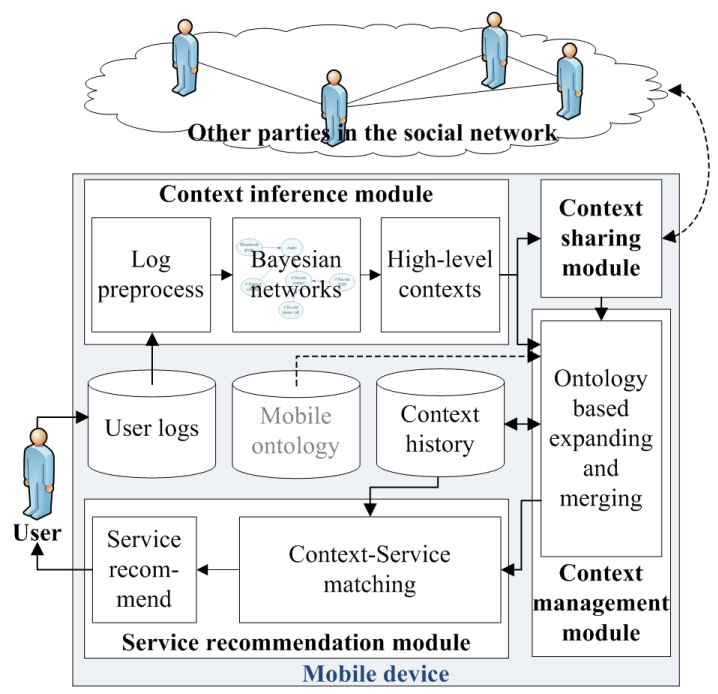

Figure 1. The overall architecture of the proposed system

\subsection{Social and personal context modeling}

The propose method firstly collects user logs from the mobile device, and performs preprocessing before the logs are used for BNs. Table 1 shows the logs and their preprocessed values. Here, the coordinates from GPS logs are used to extract location information. In this paper, we divided the domain area with lattice and then labeled each region.

The proposed method infers three kinds of highlevel contexts from the mobile logs using BNs: a social context and two personal contexts. Amity between the user and the other party in the social network is considered as the social context while the user's emotion and degree of busyness are inferred as the personal contexts. Since the user's emotion is complicated and difficult to extract, we simplified it as one of the four types of emotions on valence-arousal space [8].
Table 1. User logs collected from the mobile device

\begin{tabular}{ll}
\hline Mobile log & Value \\
\hline Time & $\begin{array}{l}\text { Morning, noon, afternoon, } \\
\text { evening, night, dawn }\end{array}$ \\
Day of week & Beginning, middle, end \\
Location (GPS) & Latitude, longitude \\
Type of schedule (PIMS) & Business, friendship, none \\
Phonebook group & Business partner, friend, lover \\
\# Schedule & Many, few \\
Anniversary & Yes, no \\
\# Recent call & Many, few \\
\# Recent SMS & Many, few \\
\hline
\end{tabular}

Let $G\left(B_{s}, \theta\right)$ be the $\mathrm{BN}$ model where $B_{s}$ and $\theta$ denote the network structure and the set of network parameters, respectively. $\theta$ is composed of the conditional probability table $B_{\Phi}$ and the prior probability distribution $B_{p}$. For the prior probability $P(\theta)$, the knowledge discovery process is as follows:

$$
P\left(Z_{T}, Y_{T}, \theta\right)=P\left(Y_{T} \mid Z_{T}, B_{\Phi}\right) P\left(Z_{T} \mid B_{p}\right)
$$

where $Z_{T}=\left\{z_{1}, z_{2}, \ldots, z_{T}\right\}$ represents a set of $T$ states variables, and $Y_{T}$ is the corresponding observations. In this paper, we have specified $B_{s}$ and $\theta$ manually based on domain knowledge. Figure 2 shows the BNs used to infer the contexts.

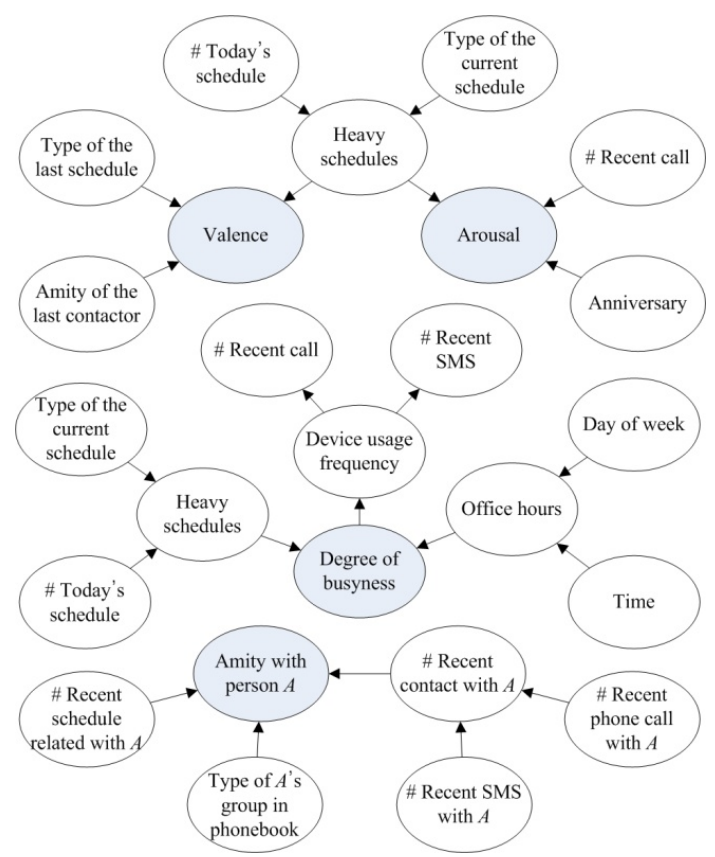

Figure 2. Bayesian networks used to infer contexts

\subsection{Context-service matching}

In addition to the three kinds of high-level contexts, four kinds of low-level contexts such as time, day of week, location, and schedule are also considered in 
order to model the user's service consuming tendency. The proposed method expands each context to a context-tree based on domain-ontology after the contexts are shared or synchronized with other devices' on the social network where the user belongs to. Figure 3 illustrates an example of the context-tree expansion procedure in case of the schedule context. The number on each node represents the frequency of the node. Leaf nodes represent the context values, and parent nodes contain higher concepts including their child nodes. When the user makes a phone call or consumes a service, his/her past contexts are accumulated as the frequency of the corresponding leaf nodes. The frequency of the parent node is then calculated by summing its children's. All other contexts are also expanded and updated in the same way.

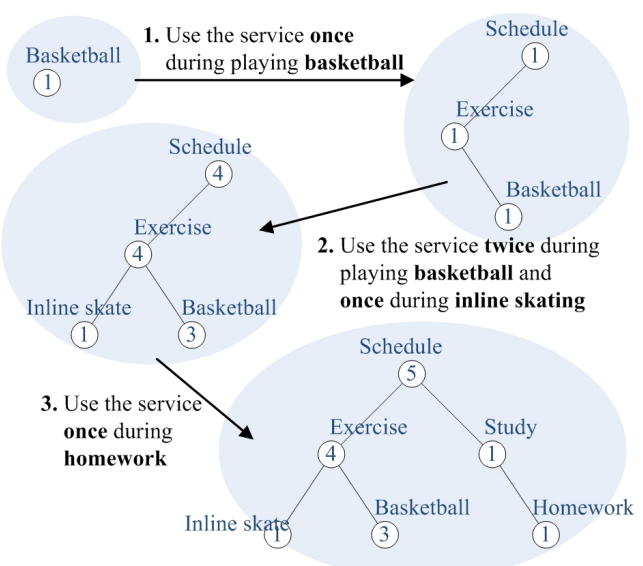

Figure 3. Expansion of context-tree

In order to recommend proper services for the user's current situation, the user's current contexts are compared with the context-history. Let $s$ be a service, $c$ be the kind of context, and $T_{H(s), c}$ be the corresponding history-tree. The similarity between $T_{H(s), c}$ and the current context-tree $T_{R, c}$ is computed as follows:

$$
\begin{gathered}
\operatorname{Sim}\left(T_{H(s), c}, T_{R, c}\right)=\sum_{i=1}^{\left|T_{H(s), c}\right|} \operatorname{Ind}(i) v(i) f(i) / \sqrt{f(\text { root })} \\
\operatorname{Ind}(i)=\left\{\begin{array}{l}
0 \text { if } T_{H(s), c}(i) \neq T_{R, c}(j) \\
1 \text { otherwise }
\end{array}, j=1, \ldots,\left|T_{R, c}\right|\right.
\end{gathered}
$$

where $v(i)$ and $f(i)$ denote level and frequency of the $i$ th node in $T_{H(s), c}$, respectively. The level, which makes possible to compare the contexts of similar concepts, increases from 0 (root node's level) to the depth of the tree.

Once the similarities are estimated for all kinds of contexts, the fitness score of the service $s$ is calculated as follows:

$$
\operatorname{Score}(s)=\sum_{c} w_{c} \operatorname{Sim}\left(T_{H(s), c}, T_{R, c}\right)
$$

where $w_{c}$ denotes the importance of $c$. Here, $w_{c}$ should be decided differently according to the application domain. Finally, the service of highest score

$$
s_{\text {best }}=\arg \max _{\mathrm{s}}(\operatorname{Score}(s))
$$

is recommended to the user.

\section{Implementations and experiments}

When the user wants to make a phone call using the mobile phone, the user has to find someone who he/she wants to contact by accessing the phonebook application sequentially (searching for each item) or manually (typing opponent's name) which bothers the user. The user also can use speed dials, but it is difficult to remember all of them. Sometimes, the user would forget to contact to someone who he/she has an appointment with or has not met recently.

In order to provide a convenient way to make phone call, we have implemented a smart phonebook application that recommends the other party's phone numbers based on the user's current situation. The smart phonebook sorts the recommended list according to the fitness scores which are shown as the icons of colored block beside the corresponding items. When the user selects an item, he/she can confirm the reason of recommendation at the below text box. It is provided as text strings based on the contexts of higher similarity among the current situation using simple rules. Figure 4 shows the prototype of the application implemented on the MS Windows Mobile 2003 SE Pocket PC platform and the recommendation scenarios for the calling situations.

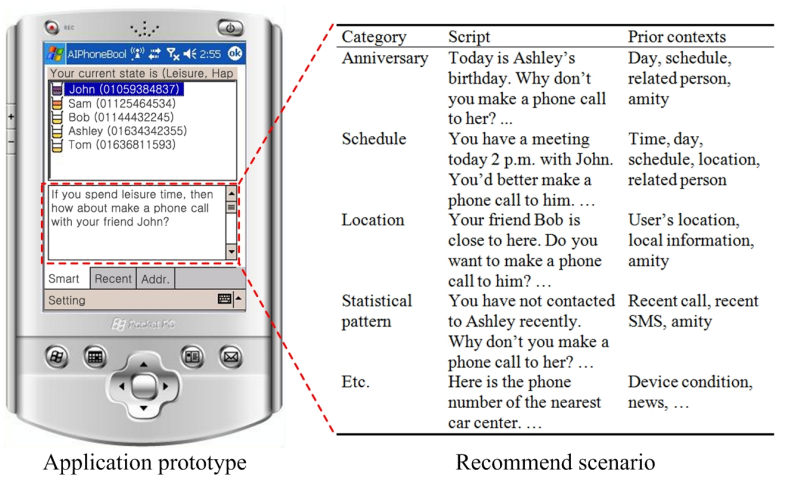

Figure 4. Prototype of the smart phonebook

Experiments on real user data have been performed to validate the proposed method. In this paper, we considered a user with the smart phonebook because it is difficult to gather the logs of several users interact 
with each other on real world environments. We have collected the logs of a university student over 12 days using Samsung Anycall SPH-M4650 smart phone device. There have been 42 received calls (including 2 missed calls) and 23 sent calls where 28 phone numbers were registered to the phonebook.

As shown in Figure 5, the user made a phone call with the first candidate for the $26.1 \%$ of calling events, while the application failed to recommendation for $21.7 \%$ because of the first phone calls with the receivers. When the application recommended five candidates, the accuracy was increased by $69.6 \%$.

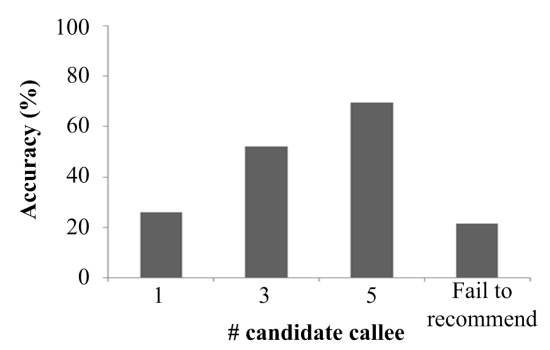

Figure 5. Accuracy of the contact list recommendation

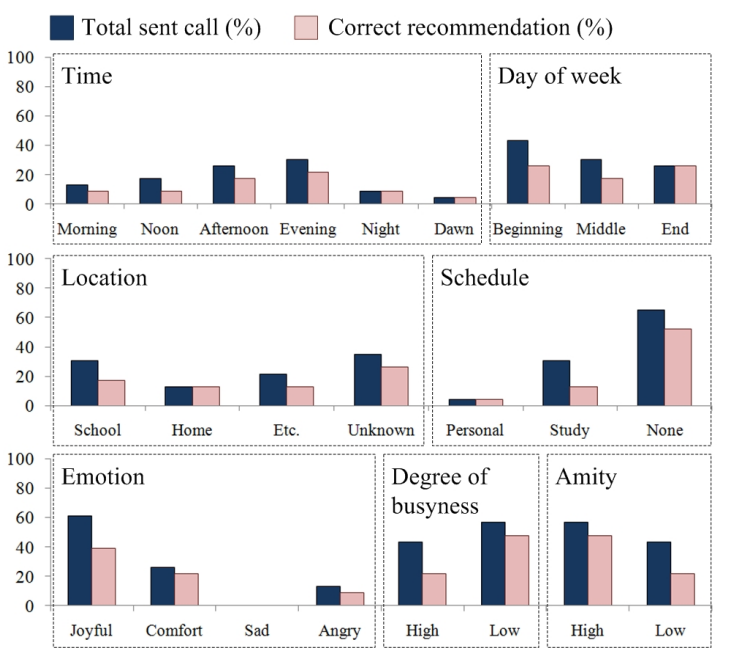

Figure 6. The distribution of the user's calling pattern and recommendation accuracy for each situation

Figure 6 illustrates the distribution of the user's calling pattern and recommendation accuracy for each situation. The application showed good performance on overall situations. In case of the schedule context, however, the recommendations were not accurate due to the insufficient logs. When the user was busy, it was difficult to estimate the user's tendency since there were many personal and social factors changing rapidly. The first phone calls with the receivers of low amity also difficult to recommend. The application records the logs for every phone call event and updates the context-history. The recommendation accuracy, therefore, could be increased as more context-history is accumulated.

\section{Conclusions}

This paper presented a social and personal context modeling method on mobile device. It infers the user's contexts from uncertain logs of mobile device using Bayesian networks and selects proper services according to the user's service consuming history. We estimated the history as context-trees and matched them in a hierarchical manner to treat the fuzziness of context information more effectively. Based on the proposed method, we have implemented a smart phonebook application which recommends contact list based on the user's situation. Experimental results on the real-user data with the application showed that the method provides an efficient and accurate means for mobile based intelligent services. As a future work, we will investigate these problems on the social networking environment where the users interact and share their mobile contexts each other.

Acknowledgement. This research was supported by Samsung Electronics Co. Ltd.

\section{References}

[1] B. Shevade, H. Sundaram, and L. Xie, "Exploiting personal and social network context for event annotation," Int. Conf. on Multimedia and Expo, pp. 835-838, 2007.

[2] J.I. Khan and S.S. Shaikh, "Computing in social networks with relationship algebra," J. of Network and Computer Applications, vol. 31, no. 4, pp. 862-878, 2008.

[3] H. Lee, J. Park, E. Ko, and J. Lee, "An agent-based context-aware system on handheld computers," Int. Conf. on Consumer Electronics, pp. 229-230, 2006.

[4] N. Sadeh, E. Chan, and L. Van, "MyCampus: An agentbased environment for context-aware mobile services," Int. Joint Conf. on Autonomous Agents and Multi-Agent Systems, pp.152-158, 2003.

[5] M. Raento, A. Oulasvirta, R. Petit, and H. Toivonen, "ContextPhone: A prototyping platform for context-aware mobile applications," IEEE Pervasive Computing, vol. 4, no. 2, pp. 51-59, 2005.

[6] M.-H. Park, J.-H. Hong, and S.-B. Cho, "Location-based recommendation system using Bayesian user's preference model in mobile devices," Int. Conf. on Ubiquitous and Intelligence Computing, pp. 1130-1139, 2007.

[7] S.-B. Cho, K.-J. Kim, K.-S. Hwang, and I.-J. Song, "AniDiary: Daily cartoon-style diary exploits Bayesian networks," IEEE Pervasive Computing, vol. 6, no. 3, pp.6675, 2007.

[8] J.A. Russel, M. Lewicka, and T. Niit, "A cross-cultural study of a circumplex model of affect," $J$. of Personality and Social Psychology, vol. 57, pp. 848-856, 1989. 